\title{
Ramos Arenas, Fernando: Cinephilie unter der Diktatur. Filmkultur in Spanien und der DDR in den 1950er und 1960er Jahren.
}

\author{
Berlin: J. B. Metzler 2021. 479 Seiten. Preis: $€ 69,99$
}

\section{Thomas Wiedemann}

Angenommen: 22. Dezember 2021 / Online publiziert: 10. Januar 2022

(C) Der/die Autor(en) 2022

Die Forschung zur Medien- und Kommunikationsgeschichte in der DDR hat in der deutschsprachigen Kommunikationswissenschaft einen festen Platz, für Arbeiten zum Film bzw. zur Filmgeschichte lässt sich das leider nicht behaupten. Die von Fernando Ramos Arenas vorgelegte Analyse der Filmkultur in der DDR und in Spanien zur Zeit des Neuen Kinos, eine überarbeitete Fassung der Habilitationsschrift des Autors an der Universität Leipzig 2020, stellt für beide Forschungsgebiete gewiss eine Bereicherung dar. Einerseits aufgrund ihres Fokus auf Rezeptionsprozesse hinsichtlich des Mediums Film in der DDR sowie der komparativen Perspektive, andererseits wegen ihrer Beschäftigung mit der Peripherie in Sachen Filmgeschichte, mit zwei Ländern fernab der westeuropäischen Zentren, die bei der Ergründung des inter- und transnationalen Phänomens der Cinephilie bis dato kaum Thema waren.

Das Unterfangen, Transformationen der Filmkultur oder noch genauer die Genese und Entwicklung eines spezialisierten Subfeldes, das kulturelle Alternativen zu den von politischen Maßgaben durchdrungenen nationalen Filmlandschaften präsentierte, vergleichend an den Beispielen Spanien und DDR in den 1950er und 1960er Jahren zu untersuchen, ist natürlich hochinteressant. Denn beide Länder bzw. Regierungsformen - von Ramos Arenas klar als Diktaturen benannt - kennzeichnete eine restriktive Kulturpolitik (,,autoritär“ in Spanien mit Zensur und Repression, „totalitär“ in der DDR mit Kontrolle, aber vor allem Steuerung durch Staat und Partei; S. 24), sodass es dort mit filmkultureller Autonomie und länderübergreifendem Austausch (beides Wesensmerkmale der cinephilen Traditionen etwa in Frankreich, Italien und Großbritannien) doch eigentlich nicht allzu weit her gewesen sein dürfte. Das Ziel der Arbeit, den Forschungsstand zur Geschichte der europäischen Film-

\footnotetext{
Dr. Thomas Wiedemann $(\bowtie)$

Institut für Kommunikationswissenschaft und Medienforschung, Ludwig-Maximilians-Universität München, Oettingenstr. 67, 80538 München, Deutschland

E-Mail: thomas.wiedemann@ifkw.lmu.de
} 
kultur zu differenzieren, also „Beispiele aufzuzeigen, Traditionen aufzudecken, Zusammenhänge zu erkennen, die unter anderen kulturpolitischen Kontexten zustande kamen, und somit gängige Annahmen der Filmgeschichtsschreibung zu korrigieren oder zu ergänzen“" (S. 5), hätte insofern durchaus noch selbstbewusster formuliert werden können.

Eine große Stärke der Studie ist der schon in der Einleitung ausgeflaggte theoretische Rahmen, namentlich die Kunstsoziologie Bourdieus und das Feld der kulturellen Produktion, dessen Charakteristika auf das filmkulturelle Feld bzw. die Strukturen und Logik eines cinephilen Subfeldes der eingeschränkten Produktion übertragen werden - Ausdifferenzierungsprozesse und limitierte Autonomie (hier gegenüber der politischen Macht) rücken dadurch automatisch in den Mittelpunkt. Mit Gewinn leitet Ramos Arenas aus der Theorie dann auch die Untersuchungsdimensionen „Institutionen“ (institutionelle Entwicklungen), ,Akteure“ (Herkunft und Aktivitäten der Cineasten) und „Diskurse“ (Aushandlungen der Filmkultur insbesondere in Zeitschriften) ab, auch wenn er darauf verzichtet, die sich daraus ergebenden Analysekategorien in ihrer Gänze zur Diskussion zu stellen. Vermisst wird in der sonst so umsichtigen Darlegung des konzeptionellen Rahmens der Studie und des Forschungsstandes aber vor allem eine Bezugnahme auf die kommunikationswissenschaftliche Beschäftigung mit Öffentlichkeitsstrukturen in der DDR.

Gestützt auf eine Vielzahl an Quellen (Archivmaterial, Zeitzeugeninterviews, Fachperiodika) und mithilfe der aus der Forschung zur klassischen Cinephilie stammenden „strukturierenden Achsen“ Raum, Zeit und Status gelingt dem Autor die Rekonstruktion eines lebendigen Subfeldes der Filmkultur in Spanien und der DDR, das trotz politischer und materieller Einschränkungen bis zu einem gewissen Grad erfolgreich nach Autonomie strebte. So erreichte auch die zwei untersuchten Länder ab Mitte der 1950er Jahre eine filmkulturelle Erneuerungsbewegung, die vor allem in Form von Filmklubs, inoffiziellen filmkulturellen Netzwerken und kritischen Beiträgen zum Fachdiskurs Begeisterung für das Medium Film zu entfachen sowie ein neues Verständnis von Kino zu pflegen und zu verbreiten versuchte. In beiden nationalen Kontexten wurden die vielen Initiativen in einer Nische geduldet (auch weil sie sich, im Fall der DDR, mit den kulturpolitischen Aktivitäten des Staates überschnitten), wenngleich dies, wie das Beispiel Spanien zeigt, zulasten ihrer gesellschaftlichen Relevanz ging. Begleitet von einer Periode der relativen Öffnung der Kulturwelt bewegten sich die Praktiken im cinephilen Feld, das intensiv in die Vergangenheit blickte und dezidiert international orientiert war, Anfang der 1960er dann weg vom filmvermittelnden Impuls in Richtung Ausdifferenzierung und Abgrenzung, ehe die fortschreitende Spezialisierung im Zuge der abklingenden Liberalisierung zum Ende des Jahrzehnts mit Repression quittiert wurde (folgenreich in der DDR insbesondere das 11. Plenum des ZK der SED). Dass die vergleichende Untersuchung wie vom Autor postuliert neben der Suche nach Gemeinsamkeiten und Systematisierung auch Platz für Unterschiede, heterogene Resultate und Einzelfälle lässt, macht vor allem das Kapitel ,Alternative Perspektiven: Alltag der Filmklubs und cinephile Akteure" deutlich, welches die Arbeit an der Basis (Filmklubs in Barcelona und Leipzig) exemplarisch beleuchtet und Protagonisten der Bewegung (fast ausschließlich junge Männer) detailreich porträtiert. 
Cinephilie unter der Diktatur wird damit dem eigenen Anspruch gerecht und bedeutet für Forschende zur europäischen Filmgeschichte hoffentlich mehr als nur einen weiteren Eintrag in der Literaturliste. Doch auch für kommunikationswissenschaftliche Film-Laien verspricht die materialgesättigte Studie eine spannende und gehaltvolle Lektüre mit vielen Anknüpfungspunkten. Den Transfer ins Fach herzustellen wäre allemal lohnend.

Funding Open Access funding enabled and organized by Projekt DEAL.

Open Access Dieser Artikel wird unter der Creative Commons Namensnennung 4.0 International Lizenz veröffentlicht, welche die Nutzung, Vervielfältigung, Bearbeitung, Verbreitung und Wiedergabe in jeglichem Medium und Format erlaubt, sofern Sie den/die ursprünglichen Autor(en) und die Quelle ordnungsgemäß nennen, einen Link zur Creative Commons Lizenz beifügen und angeben, ob Änderungen vorgenommen wurden.

Die in diesem Artikel enthaltenen Bilder und sonstiges Drittmaterial unterliegen ebenfalls der genannten Creative Commons Lizenz, sofern sich aus der Abbildungslegende nichts anderes ergibt. Sofern das betreffende Material nicht unter der genannten Creative Commons Lizenz steht und die betreffende Handlung nicht nach gesetzlichen Vorschriften erlaubt ist, ist für die oben aufgeführten Weiterverwendungen des Materials die Einwilligung des jeweiligen Rechteinhabers einzuholen.

Weitere Details zur Lizenz entnehmen Sie bitte der Lizenzinformation auf http://creativecommons.org/ licenses/by/4.0/deed.de.

Dr. Thomas Wiedemann ist wissenschaftlicher Mitarbeiter am Institut für Kommunikationswissenschaft und Medienforschung der Ludwig-Maximilians-Universität München. 